\title{
Long-Term Enhancement of Hippocampal Synaptic Transmission and the Acquisition of Spatial Information
}

\author{
B. L. McNaughton, C. A. Barnes, G. Rao, J. Baldwin, and M. Rasmussen \\ Department of Psychology, University of Colorado, Boulder, Colorado 80309
}

\begin{abstract}
The hypothesis that memories are stored as a specific distribution of strengths in a population of modifiable synapses was examined by the bilateral induction of long-term enhancement in synapses of the main afferent fiber system to the hippocampal formation in rats. Brief, high-frequency activation of the perforant pathway in chronically prepared animals resulted in a persistent increase in the field EPSP and population spike, measured extracellularly in fascia dentata. This treatment resulted in a profound and persistent deficit in the acquisition of new spatial information in a task requiring spatial "reference" memory, and disruption of recently acquired spatial information. Well-established spatial memory was completely unaffected, however, as was the acquisition of spatial information into shortterm "working" memory. These results support the hypothesis that, during the formation of "cognitive maps," spatial information must be temporarily stored at modifiable synapses at the input stage to the hippocampal formation, but that this information is not needed once the representation of the environment is well established. Spatial working memory, in a familiar environment, appears not to depend on the distribution of synaptic strengths in this system at all.
\end{abstract}

Most attempts to explain associative learning in the nervous system have invoked long-term changes in the efficacy of cellto-cell communication as the underlying information-storage medium (e.g., Hebb, 1949; Marr, 1971). The common element of these theories is the assumption that information is stored as a specific distribution of modifiable synaptic weighting functions. These hypotheses predict that a treatment that disrupts such a distribution, by driving the population of modifiable synapses to the maximum strength, will disrupt the information content. This would have two consequences: impaired performance of tasks requiring the integrity of previously stored information, and impaired acquisition of new information. The present experiments were designed to examine this prediction by studying the effects on spatial learning and memory of artificially induced enhancement of synaptic efficacy in a large population of hippocampal synapses.

Bliss, Lomo, and Gardner-Medwin first documented that brief episodes of electrical stimulation of perforant path fibers at physiological frequencies resulted in a lasting increase in synaptic transmission to hippocampal target neurons (Bliss and GardnerMedwin, 1973; Bliss and Lomo, 1973). Since this discovery, considerable evidence has accumulated in support of the hypothesis that this experimentally induced phenomenon represents the activation of a physiological process that normally

\footnotetext{
Received Apr. 5, 1985; revised July 15, 1985; accepted July 17, 1985.

This work was supported by PHS Grants AG03376 and NS20331. We thank Seth Sharpless for help with computer software, B. Peterson for secretarial assistance, and $P$. Sharp and B. Jones Leonard for helpful comments on the manuscript.

Correspondence should be sent to B. L. McNaughton, Department of Psychology, University of Colorado, Campus Box 345, Boulder, CO 80309.

Copyright (c) 1986 Society for Neuroscience $0270-6474 / 86 / 020563-09 \$ 02.00 / 0$
}

subserves associative-information storage. Although this phenomenon was originally called "long-lasting potentiation" by Lomo (1966), and the term LTP (long-term potentiation) is in common use, it is now clear that this mechanism and its properties are fundamentally different from the forms of "potentiation" that have been studied extensively in experiments at both neuromuscular and central synapses (Birks, 1977; del Castillo and Katz, 1954; Liley and North, 1953; Lloyd, 1949; Magleby and Zengel, 1975). McNaughton et al. (1978) demonstrated that, unlike potentiation, this long-lasting phenomenon rcquircd the cooperative activity of multiple inputs to common postsynaptic elements, and therefore introduced "long-term enhancement" (LTE) as an alternative term. This distinction was supported by the subsequent demonstration that LTE and post-tetanic potentiation can occur independently at the same hippocampal synapses, and do not share a common mechanism (McNaughton, 1980).

In addition to its associative character, a number of other properties of LTE are consistent with the hypothesis that it plays a role in information storage. LTE is specific to the synapses involved in high-frequency convergence events (Andersen et al., 1977; Levy and Steward, 1979; McNaughton and Barnes, 1977). Its persistence is increased by stimulus repetition (Barnes, 1979), and its decay is correlated with behaviorally assessed forgetting of place information (Barnes, 1979; Barnes and McNaughton, 1985a). The characteristics of LTE in relation to its possible role in information storage have recently been reviewed (Barnes and McNaughton, 1985b; McNaughton, 1983; Swanson et al., 1982; Teyler and Discenna, 1984).

The relationship of LTE in hippocampal synapses to spatial learning and memory was chosen for study because there is clear and abundant evidence that spatial information-processing is at least one, if not the primary, function of the hippocampal formation (O'Keefe and Nadel, 1978), and it is a common element of most recent theories of hippocampal function. O'Keefe and Nadel (1978), for example, maintain that the hippocampus is the neural substrate of a cognitive mapping system. Olton and his colleagues (Olton, 1983; Olton et al., 1979), on the other hand, contend that the hippocampus plays a crucial role in shortterm working memory of all kinds, including the short-term storage of information about recently visited locations. Marr (1971) proposed that the hippocampus is an intermediate-term simple associative memory system that stores representations of all recent experience until other parts of the nervous system can classify this information for appropriate storage in longterm memory. This information, particularly in an animal such as a rat, would have a large spatial component. Squire and Cohen (1984; Squire et al., 1984) suggest that the hippocampus is involved in the formation and consolidation of declarative memory. This includes memory of places and the events that occurred there. In their hypothesis, the hippocampus serves to maintain relationships among cortical structures until long-term associations can be established there. A somewhat similar hypothesis, 


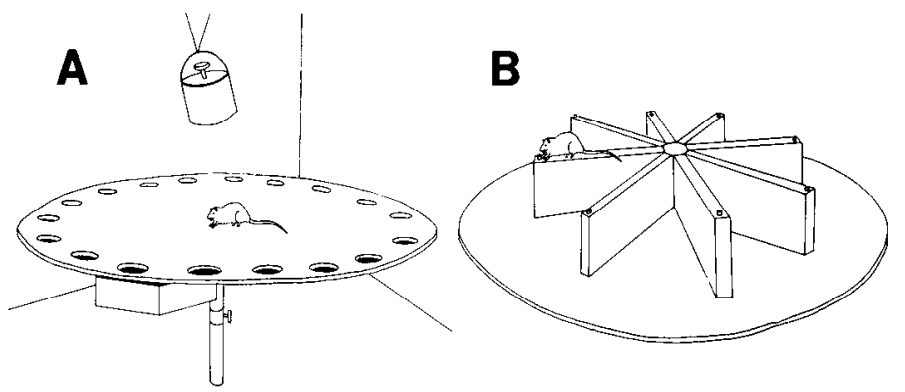

Figure 1. A, Drawing of the circular platform apparatus for the investigation of spatial-reference learning and memory. The animals' task was to escape from the brightly illuminated surface into a dark goal tunnel located under one of 18 holes surrounding the perimeter. At the start of each trial, animals were placed in a random orientation into a false-bottomed, cylindrical start chamber, which was then raised by a system of pulleys, leaving the animal free to move on the maze surface and thus preventing the use of fixed motor sequences to solve the task. The maze surface was rotated randomly from trial to trial, preventing the use of odor trails or other intramaze cues to solve the task. The goal tumel was held in a fixed location, relative to the extramaze cues, from trial to trial but could be shifted to a new location to assess the animal's ability to alter its established representation of spatial relationships. $B$, Drawing of the radial eight-arm maze used to assess the acquisition and retention of trial-specific working memory information. This task requires that animal recover the food from the end of each of the eight arms without reentry into an arm from which the food has already been taken. Numerous studies have shown that rats tend not to use motor sequences to optimize their performance, but rather remember the locations of the places they have recently visited.

proposed by Teyler and Discenna (in press), suggests that the hippocampus serves as an indexing system, which stores the addresses of recently activated cortical elements. Gabriel and colleagues (Gabriel et al., 1985) propose that the hippocampus provides crucial contextual information during the formation of conditioned responses. In their view, this information controls a gating mechanism that determines whether sensory input will have access to motor output systems. Perhaps the most important contextual information would be spatial in nature. Recently, Rawlins (1985) proposed that the hippocampus stores information of all types and is necessary whenever the bridging of temporal gaps in experience is required. Rawlins maintains that place learning involves the formation of temporally discontiguous associations during exploration, and is hence susceptible to hippocampal disruption.

In addition to these theories, which are based primarily on the effects of hippocampal damage, several types of neurophysiological data suggest a major spatial involvement in hippocampal function: single units in the hippocampus and associated structures show strong spatial determinants in their firing properties (Kubie and Ranck, 1983; McNaughton et al., 1983; O'Keefe, 1976; O'Keefe and Dostrovsky, 1971; Olton et al., 1976; Ranck, 1984); the persistence of hippocampal LTE shows a significant correlation with performance on a spatial-reference memory task where animals are required to remember one specific location from trial to trial (the circular platform, described below; Barnes, 1979); and finally, persistent changes in synaptic input/output relations in the primary cortical afferent pathway to the hippocampus result from exposure of animals to novel complex environments (Sharp et al., 1985).

The experiments presented here address the specific question of whether, and how, spatial information from the neocortex is stored at the primary input stage to the hippocampus. Some of these results have been described earlier in abstract form (Barnes and McNaughton, 1983; Rao et al., 1984).

\section{Materials and Methods}

The data presented here are the results of four experiments carried out over a 3-year period. The subject pool contributing to these data consisted of 62 male Fischer 344 retired breeder rats, ranging in age from 8 to 10 months at the beginning of the experiment. These animals were obtained from Charles River laboratories. The animals were maintained on a 12-12 hr light-dark cycle, and all behavioral testing was carried out in the last quarter of the light cycle.

\section{Behavioral apparati}

Two types of behavioral apparatus were used in these studies. Experiments 1, 2 and 3 employed the circular-platform, spatial-reference mcmory problem, which was first described by Barnes (1979). The apparatus for this task (Fig. 1A) consisted of a white circular platform, $1.2 \mathrm{~m}$ in diameter, around the circumference of which were 18 equally spaced 9 $\mathrm{cm}$ diameter holes. The maze surface could be rotated independently of a dark goal tunnel located beneath it. Trials were started with the animal being placed into a cylindrical start chamber with a false bottom. This chamber rested in the center of the maze, and could be raised to the ceiling by a system of pulleys (Fig. 1 $A$ ), leaving the animal free to move about the surface. The maze surface was brightly illuminated by three $150 \mathrm{~W}$ spotlights located directly above the maze. The maze surface orientation, relative to the goal tunnel, was randomized from trial to trial, as was the orientation of the animals when placed into the start chamber. In Experiments 1, 2, and 3A, the circular platform resided in a $5.6 \times 3.7 \mathrm{~m}$ room with numerous distinguishing features on the walls surrounding the maze. During Experiment $3 \mathrm{~B}$, the maze was transferred to an eight-sided, $3.2 \mathrm{~m}$ diameter enclosure, which was located in a different room. Seven of the eight walls possessed a distinct visual cue, and outside but adjacent to one of these walls was the sound of a computer in operation.

Two types of scores were obtained: the number of holes erroneously investigated, and the angular deviation from the goal-tunnel location of the first hole investigated (measured in units of number of holes away from the correct one). An error was scored if the animal's nose dropped below the maze surface into an incorrect hole. Repetitive errors in the same hole were counted only if the animal moved its whole body away from the hole before reinvestigating it. Escape from the brightly illuminated surface provided a high degree of motivation, as indicated by the fact that, in the 2172 trials in these cxpcriments, there were no instances where a rat failed to enter the tunnel immediately upon locating it, nor were there any instances where animals returned to the maze surface from the tunnel. Nevertheless, in Experiment 3B, in which animals were given four trials per day, added incentive was provided by restricting the animals' water access to a $3 \mathrm{hr}$ period following the daily test sessions, and by placing $0.1 \mathrm{~cm}^{3}$ of a concentrated sugar solution into a cup located at the back of the goal tunnel.

The second behavioral task (used in Experiment 4) consisted of a well-studied working memory problem on an elevated, radial eight-arm maze (Olton and Samuelson, 1976; Fig. 1B). The maze was located in a $3.1 \times 3.1 \mathrm{~m}$ room, which was illuminated by a single $25 \mathrm{~W}$ bulb located on one wall. The central platform was $14 \mathrm{~cm}$ in diameter, and the arms were $62 \mathrm{~cm}$ long, $5.4 \mathrm{~cm}$ wide, and $30 \mathrm{~cm}$ high. Foodcups were located at the cnds of cach arm for the delivery of reinforcement, which consisted of either chocolate milk $\left(0.2 \mathrm{~cm}^{3} / \mathrm{arm}\right)$, or a mixture of grated apples, butter, and ground rat chow (approximately $0.2 \mathrm{~cm}^{3 /}$ arm).

For the radial-maze experiment, animals were maintained at $80-85 \%$ of their free-feeding weights by restricting access to food. The animal's task in this experiment was to retrieve the food from all eight arms without reentering arms from which the food had already been consumed. An error was scored when the animal went more than two-thirds of the way down a previously visited arm.

\section{Surgical procedures}

Animals were deprived of food and water for $12 \mathrm{hr}$ prior to surgical anesthesia, which was induced by an intraperitoneal injection of sodium pentobarbital $(20 \mathrm{mg} / \mathrm{kg})$. The initial anesthetic was supplemented, if necessary, by small doses of sodium pentothal. In some animals chloral hydrate was used as a supplemental anesthetic; however, this practice was discontinued because of a relatively high incidence of postsurgical gastrointestinal disorder. No animals with a serious gastrointestinal disorder are included in the results reported here. 


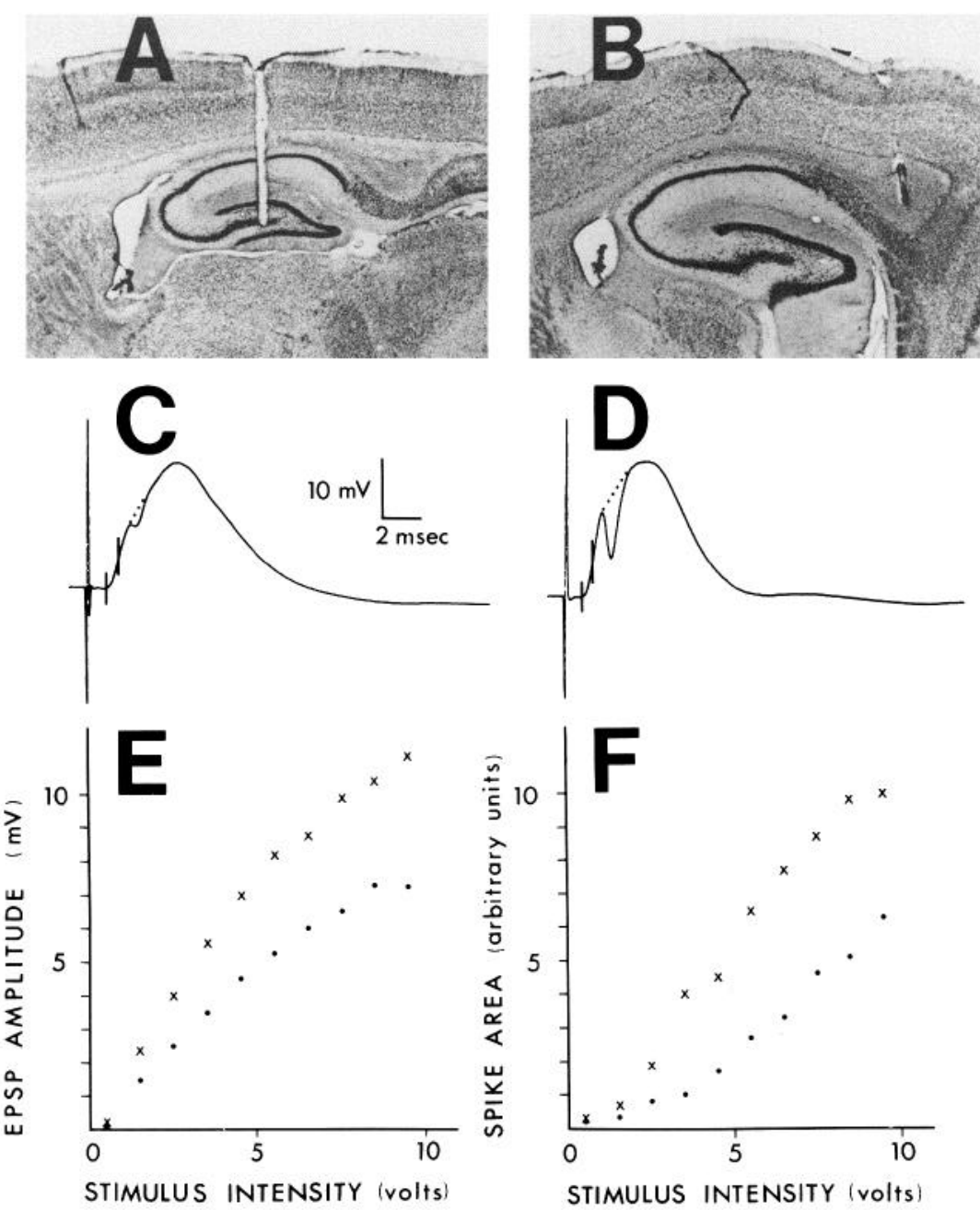

Figure 2. $A$ and $B$, Examples of histological reconstructions showing the typical location of the recording electrode $(A)$ in the fascia dentata, and of the stimulating electrode $(B)$ in the white matter of the angular bundle, where perforant path fibers converge en route to the hippocampus from the entorhinal cortex. $C$ and $D$, Examples of evoked responses recorded in fascia dentata following single-pulse activation of perforant pathway at a stimulus intensity of $2.5 \mathrm{~V}$. The trace in $C$ was recorded prior to high-frequency stimulation, whereas the trace in $D$ was recorded $5 \mathrm{~d}$ following the last high-frequency session. The amplitude of the synaptic component was assessed by taking the voltage difference between EPSP onset and $0.5 \mathrm{msec}$ after onset. The latter point was on the rising phase of the field EPSP prior to the onset of the population spike. The amplitude of the population spike was measured by fitting a line tangent to the response waveform at two points, corresponding approximately to the onset and offset of the spike, and calculating the area between the curve and this line. These measures are indicated above. $E$ and $F$, Examples of input/output curves relating the synaptic $(E)$ and populationspike $(F)$ response to the amplitude of the stimulus pulse. Filled circles, Measures prior to high-frequency stimulation; crosses, measures taken $5 \mathrm{~d}$ following the last high-frequency session.
After retraction of the scalp, burr holes of approximately $1 \mathrm{~mm}$ diameter were drilled bilaterally for the insertion of stimulating and recording electrodes. The coordinates, relative to Bregma, were as follows: $3.8 \mathrm{~mm}$ posterior and $2.4 \mathrm{~mm}$ lateral for the recording electrodes, and $8.1 \mathrm{~mm}$ posterior and $4.4 \mathrm{~mm}$ lateral for the stimulating electrodes. The dura was slit with a sharp needle. The electrodes consisted of $114 \mu \mathrm{m}$, Teflon-insulated, stainless steel wires which were cut at right angles with sharp scissors. For the stimulating electrode, $300-500 \mu \mathrm{m}$ of insulation were stripped from the tip. The recording electrode was lowered into the hilus of the fascia dentata (see Fig. $2 A$ ) by monitoring multiple-unit injury discharges (McNaughton, 1980). The stimulating electrode was lowered slowly, while stimulating with low-intensity pulses delivered once per $5 \mathrm{sec}$. The final depth was adjusted to give the maximum synaptic field response recorded in the fascia dentata (see Fig. $2 B$ ). The electrodes were fixed in place with dental acrylic, which was anchored to the skull by nine small stainless steel jewelers' screws. Three of the screws had wires soldered to them; these were used as ground and indifferent electrodes, and for the return of isolated stimulus pulses.

All leads from the animal were crimped to gold amphenol pins, which were mounted in a small connecting plug (Molino and McIntyre, 1972). The plug was then attached over the implant with dental acrylic, and the wound closed by suturing and cementing the remaining margins to the implant. The animals received daily intramuscular injection of penicillin for $5 \mathrm{~d}$ following surgery. A minimum of 3 weeks' recovery was allowed before electrophysiological or behavioral testing was begun.

\section{Electrophysiological procedures}

Extracellular field potentials were evoked in fascia dentata by stimulation of perforant path fibers in the region of the angular bundle (Fig. $2, A$ and $B$ ). Previous studies have shown that stimulation in this region elicits widespread activation of perforant path terminals throughout the dorsal hippocampal formation (Lomo, 1971). The recording chamber consisted of an electrically shielded box $(62 \times 62 \times 62 \mathrm{~cm})$, in which was placed a plexiglass cylinder, $20 \mathrm{~cm}$ in diameter and $25 \mathrm{~cm}$ in height. Recording and stimulating leads were connected to the animal through the miniature connecting plug, and to the electronic apparatus via a gold commutator. For recording, animals were connected to the recording lead and placed in the plexiglass cylinder, but were otherwise alert and unrestrained.

Two types of electrophysiological testing paradigm were employed to assess the synaptic and postsynaptic responses. In some parts of the study, input/output curves were recorded by delivering variable-intensity stimuli (Fig. 2, $E$ and $F$ ). Other parts of the study used a fixed stimulus intensity $(10 \mathrm{~V})$. The stimuli consisted of constant-voltage diphasic square-wave pulses ( $100 \mu \mathrm{sec} / \mathrm{half}$ cycle, $0.5-10 \mathrm{~V}$ in amplitude) delivered at $0.2 \mathrm{~Hz}$. Ten volt stimulation produced responses whose amplitude was close to the asymptote of the input/output curve (see Fig. 2, $E$ and $F$ ). The differential signals were filtered between $0.5 \mathrm{~Hz}$ and $10 \mathrm{kHz}$ (half-amplitude frequency cutoff points), amplified by a factor of 100 , and recorded by digital computer at a sampling rate of 20 points $/ \mathrm{msec}$. Examples of such evoked responses are shown in Figure 2, $C$ and $D$.

The synaptic component of the evoked response was measured as the voltage difference at two fixed latency points following the stimulus artifact. These points were selected so that the first was approximately at EPSP onset, while the second was prior to the onset of the postsynaptic population spike (see Fig. 2, $C$ and $D$ ). Previous studies (McNaughton and Barnes, 1977) have shown that such measures bear a proportional relationship to the peak amplitude of the synaptic field potential. The postsynaptic population spike was measured as the area under the tan- 


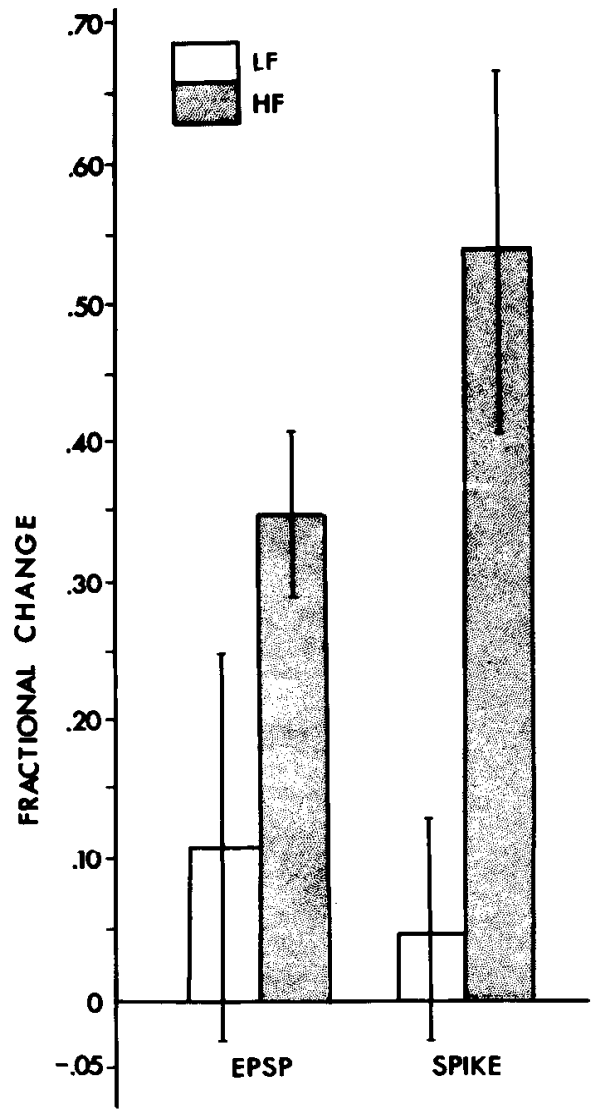

Figure 3. LTE scores for the field EPSP and population spike in the low-frequency (LF) and high-frequency (HF) groups of Experiment 1, measured relative to their baseline response amplitudes. The measures shown were recorded $24 \mathrm{hr}$ following the delivery of LTE-inducing stimulation to group $\mathrm{HF}$, and control low-frequency stimulation to group LF.

gent line joining the onset and offset of the spike (see Fig. 2, $C$ and $D$ ). Both field EPSP and population-spike measures were determined automatically by computer. In all experiments, baseline responses to lowfrequency $(0.2 \mathrm{~Hz})$ stimulation were measured both before and $24 \mathrm{hr}$ after the final LTE-inducing, high-frequency session. In some experiments, an additional recording session was carried out $5 \mathrm{~d}$ later.

Long-term synaptic enhancement was induced by delivering highfrequency stimulus bursts via the stimulation electrodes in each hemisphere. The stimulus bursts consisted of 10 pulses, with an intraburst interval of $2.5 \mathrm{msec}(400 \mathrm{~Hz})$ and an interburst interval of $5 \mathrm{sec}$. Ten bursts were delivered to each hemisphere per session. Sessions were repeated either $3 \times$ a day at $2 \mathrm{hr}$ intervals over $2 \mathrm{~d}$ ( 6 sessions total), or once per day for 12 consecutive days (Experiment $3 \mathrm{~A}$ only). In Experiment 1 , the control animals received the same total number of stimulus pulses as the high-frequency group, but the stimulus frequency was 2 $\mathrm{Hz}$. In Experiments 2-4, stimulation of control animals was at a frequency of $0.2 \mathrm{~Hz}$. Hippocampal EEG was monitored during and after all high-frequency stimulation sessions. None of the animals exhibited epileptiform afterdischarge or overt behavioral seizures at any time during the experiments.

\section{Data analysis}

The measure of LTE for both the EPSP and population spike was the fractional change in the dependent variable,

$$
\frac{V_{t}-V_{0}}{V_{0}}
$$

where $V_{t}$ is the post-high-frequency score and $V_{0}$ is the baseline measure. Statistical analysis of behavioral and electrophysiological results was carried out, using $t$ tests, with alpha set at the 0.05 level for all com- parisons. Histograms and error bars in the following figures represent means and SEM, respectively.

All animals underwent electrode implantation prior to behavioral testing. Following the experiments, the animals were perfused with a $10 \%$ Formalin solution in physiological saline. Electrode tracks were reconstructed by standard histological procedures. All recording electrodes were within or below the dorsal granule cell layer of the fascia dentata (Fig. $2 A$ ), and all stimulating electrodes were in the white matter in the region of the angular bundle (Fig. $2 B$ ). Macroscopic and histological examination of all brains revealed no sign of brain infection in any of the animals.

The detailed protocols for the individual experiments are presented in Results.

\section{Results}

\section{Experiment 1}

Purpose. This experiment was designed to assess the effects of bilateral, LTE-inducing stimulation on both memory of welllearned spatial information and on the learning of new spatial information.

Protocol and results. Twelve high-frequency (Group HF) and 12 low-frequency control animals (Group LF) were used in this study. Initial training consisted of 16 trials on the circular platform at $24 \mathrm{hr}$ intervals. This was sufficient for the animals to reach asymptotic performance. There were no between-group differences in this phase of the study, as shown in the leftmost histogram pair of Figure 4. Group HF then received bilateral LTE-inducing high-frequency stimulation over $2 \mathrm{~d}$, whereas group LF received the same total number of stimulus pulses at $2 \mathrm{~Hz}$. The resulting LTE of the synaptic and population-spike components of the evoked responses are shown in Figure 3. Group HF showed a significantly larger change in both measures compared to group LF ( $p<0.05$; see Fig. 3).

Twenty-four hours following the last stimulation session, the animals were tested again on the circular platform. On this and all subsequent trials, however, the location of the goal tunnel was rotated to a position $135^{\circ}$ from its location during initial training. This permitted an assessment of the animals' retention of the original location by considering the initial deviation from the previously correct hole, and a measurement of acquisition of the new location by considering the decrease in number of errors over trials. These data are shown in Figure 4. There was no between-group difference in the initial deviation score (see second histogram pair in Fig. 4), indicating that the retention of the original tunnel location was unaffected by high-frequency stimulation. There was, however, a large and statistically significant difference between the HF and LF groups in error scores on subsequent trials ( $p<0.05$; see Fig. 4$)$.

\section{Experiment 2}

Purpose. This experiment was designed to investigate the possibility that recently acquired spatial information might be susceptible to disruption by LTE-inducing perforant path stimulation.

Protocol and results. Eight animals were used in each of the HF and LF groups. Acquisition training was carried out as described in Experiment 1, with the exception that, within 5 min following each of the 16 daily training sessions, each animal was connected to the recording apparatus and the responses to low-frequency stimulation were recorded. Postrelocation Trial 1 followed $24 \mathrm{hr}$ after the last training session (Trial 16). On this trial, the goal location was again rotated $135^{\circ}$ from the learned location. In this study, however, the sessions of LTEinducing stimulation were initiated $5 \mathrm{~min}$ after the first exposure to the changed goal location, and again 5 min following Postrelocation Trial 2.

The LF group in this study received only $0.2 \mathrm{~Hz}$ test stimuli throughout the experiment. The HF group showed significantly 


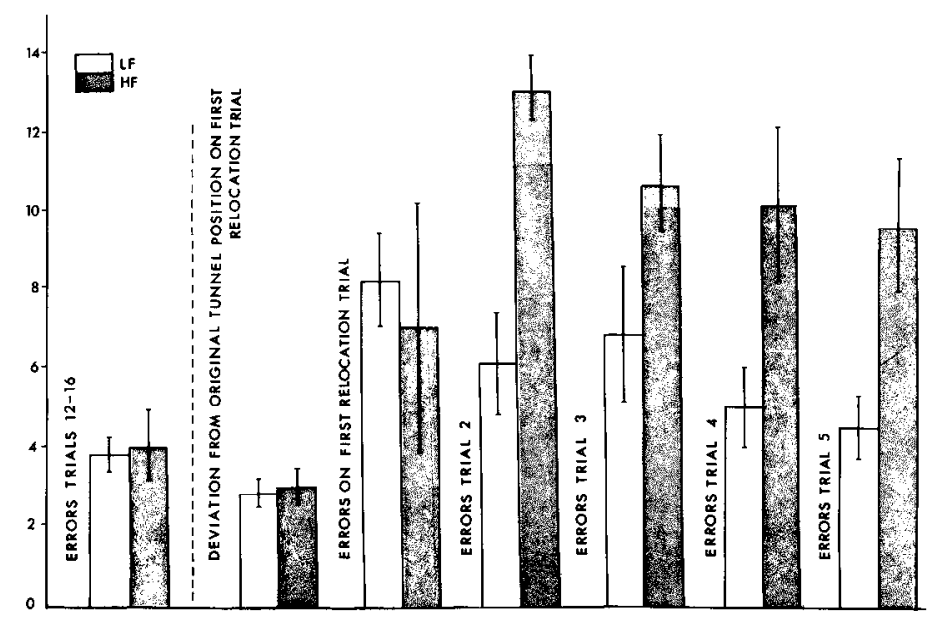

Figure 4. Effects of LTE-inducing stimulation on retention of a well-learned spatial location, and acquisition of a new spatial location (Experiment 1). The asymptotic performance during the initial acquisition (prior to high-frequency stimulation) is shown in the leftmost histogram pair. Following high-frequency (HF) and low-frequency (LF) stimulation treatments, the animals were tested on their ability to learn that the tunnel had been relocated $135^{\circ}$ from its original location. The HF and LF groups were equally unimpaired on their retention of the original tunnel position (second histogram pair), and made an equivalent number of errors in finding the new location (third histogram pair). On all subsequent trials, however, group HF exhibited a statistically significant impairment, relative to the LF group.

more LTE in both response components than the LF group (see Fig. 5).

The LF group showed a significantly smaller initial deviation from the new goal location on the circular platform than did the HF group, beginning with the second postrelocation trial $(p<0.05$; see Fig. 6). While the LF group showed continuous improvement over trials on the new task, there was no trend towards improvement by the HF group.

The significant difference on Postrelocation Trial 2 indicates that high-frequency stimulation was capable of disrupting recently acquired spatial information. Again, however, the same high-frequency stimulation failed to disrupt well-established spatial information, as indicated in Figure 7, where the same behavioral data are plotted as deviations from the original tunnel location. As can be seen in Figure 7, there was no significant difference between groups on the first trial following tunnel rotation, and the HF group maintained this initial heading throughout the remainder of the experiment, even though each animal received experience with the tunnel in its new location within each trial.

\section{Experiment 3}

Purpose. This experiment was designed to assess the effects of LTE-inducing stimulation of perforant pathway on the initial acquisition of spatial-reference memory using the circular platform task.

Protocol and results. Eleven $\mathrm{HF}$ and $11 \mathrm{LF}$ animals were used in this experiment, which was conducted in two parts. In Part $\mathrm{A}$, the HF group received high-frequency stimulation and the LF group received low-frequency stimulation once every $24 \mathrm{hr}$ for 12 consecutive days. Twenty-four hours following the last stimulation session, the animals were given their first of 16 daily training trials on the circular platform. The LF group showed statistically less change on the evoked-response measure than did the IIF group (see Fig. 8, $A$ and $B$ ). However, in this experiment, considerably less EPSP enhancement was observed than in the other studies reported here. Nevertheless, the HF group's evoked responses changed significantly over baseline. The HF group was statistically significantly different from the LF group $(p<0.05)$ in its performance on Trials 15 and 16 of the circle maze, as shown in Figure $8 C$. This difference, however, was somewhat smaller than those observed in Experiments 1 and 2.
Part B of this study was introduced to determine whether LTE-inducing stimulation affected the acquisition of new spatial information while the animals were performing a task with which they were well familiarized. The same animals used in Part A were given approximately 2 months to allow for the decay of LTE. Previous studies have shown that LTE decays with a time constant of about $37 \mathrm{~d}$ (Barnes and McNaughton, 1980). Following this recovery period, the animals received an additional 28 sessions (four sessions/d, $30 \mathrm{~min}$ intertrial interval) on the original circular platform problem (with the goal tunnel in the same location), as described in Part A. After this retraining, the circular platform itself was transferred to a second room, which differed substantially in the distribution of extramaze visual and auditory cues. For $2 \mathrm{~d}$ prior to exposure to this new test situation, the HF group received three daily sessions of LTE-inducing stimulation, whereas the LF group received the usual low-frequency test pulses. The corresponding fractional changes in the EPSP and spike are shown in Figure $9 A$.

Each animal's performance in the new situation was assessed relative to its own asymptotic performance during the retraining

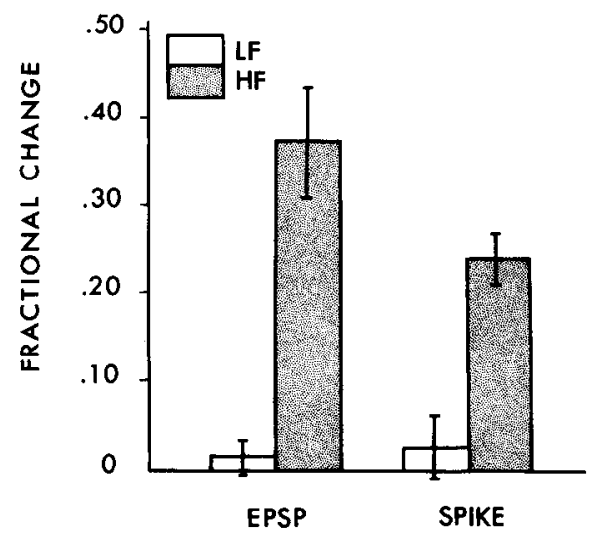

Figure 5. LTE scores for the field EPSP and population spike in the low-frequency (LF) and high-frequency (HF) groups of Experiment 2, measured relative to their baseline response amplitudes. The measures shown were recorded $24 \mathrm{hr}$ following the delivery of LTE-inducing stimulation to group $\mathrm{HF}$, and control low-frequency stimulation to group LF. 
Figure 6. Effects of LTE-inducing stimulation on recently acquired spatial information (Experiment 2). Animals were trained to asymptotic performance on the circular platform using a fixed tunnel location. The tunnel was then relocated $135^{\circ}$ from the learned position, and the animals were retrained. Within $5 \mathrm{~min}$ following Relocation Trials 1 and 2, the HF group received LTE-inducing stimulation. A significant between-group difference was obtained on Trial 2 (and all subsequent trials), indicating a disruption of information acquired during Trial 1 , and a persistent disruption in further acquisition.

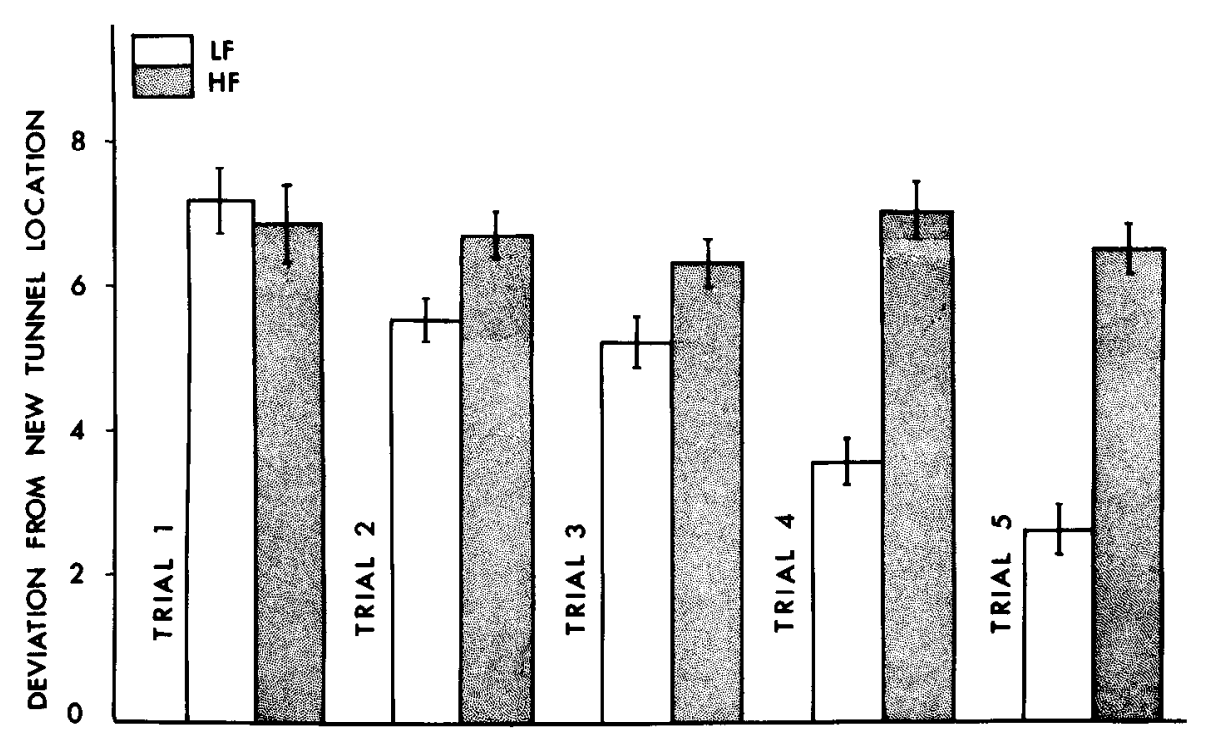

phase in the original room (mean of Trials 17-28). High-frequency stimulation produced a large and statistically significant difference in performance in the new environment compared with that of the LF group over all testing sessions (Fig. 9B). Nevertheless, unlike the results of Experiments 1 and 2, there was a clear improvement over trials in the high-frequency, as well as the low-frcquency, groups. This led to the hypothesis that the HF animals might be using a somewhat different strategy to improve their performance on this task. Previous studies using the circular platform have shown performance to be highly robust against removal of random subsets of visual cues in the testing environment (Barnes and McNaughton, 1980). This suggests that normal animals may solve the problem by forming associations among the cues in the environment, rather than depending on any single feature for task solution.

To investigate the possibility of strategy changes in the highfrequency group, the last batch of animals (four HF and four LF) was tested on two additional trials following Trial 16. On Trial 17, two visual cues were removed (those on either side of the goal tunnel). On Trial 18 , in addition to the removal of these two visual cues, the most salient auditory cue (noise from a computer adjacent to the maze enclosure) was removed.
No significant change in performance occurred in either group as a result of the removal of the visual cues on Trial 17. However, the additional removal of the auditory cue on Trial 18 resulted in a large $(102 \%)$ and statistically significant increase in the HF group's initial deviation from the correct location of the goal. There was no significant change in the LF group's behavior on Trial 18 .

\section{Experiment 4}

Purpose. This experiment examined the effects of LTE-inducing perforant path stimulation on the acquisition of information by the working memory, in a situation where the reference memory components of the task were well learned.

Protocol and results. Twelve animals, taken from the LF groups in Experiments 2 and 3, were used in this study. Animals were given a minimum of 16 training sessions on the radial eightarm maze. Eight of the animals were trained to a criterion of less than a total of six errors over 3 consecutive days, while the remaining four animals were given 21 training sessions before high-frequency stimulation. In both cases performance was approximately at asymptote.

LTE-inducing stimulation was delivered over 2 consecutive
Figure 7. Same data as shown in Fig. 6 , replotted as deviation from the original tunnel location. While highfrequency stimulation severely disrupted retention of recently acquired spatial information and prevented acquisition of new information, it had no effect on the animals' retention of the original, well-learned, tunnel location.

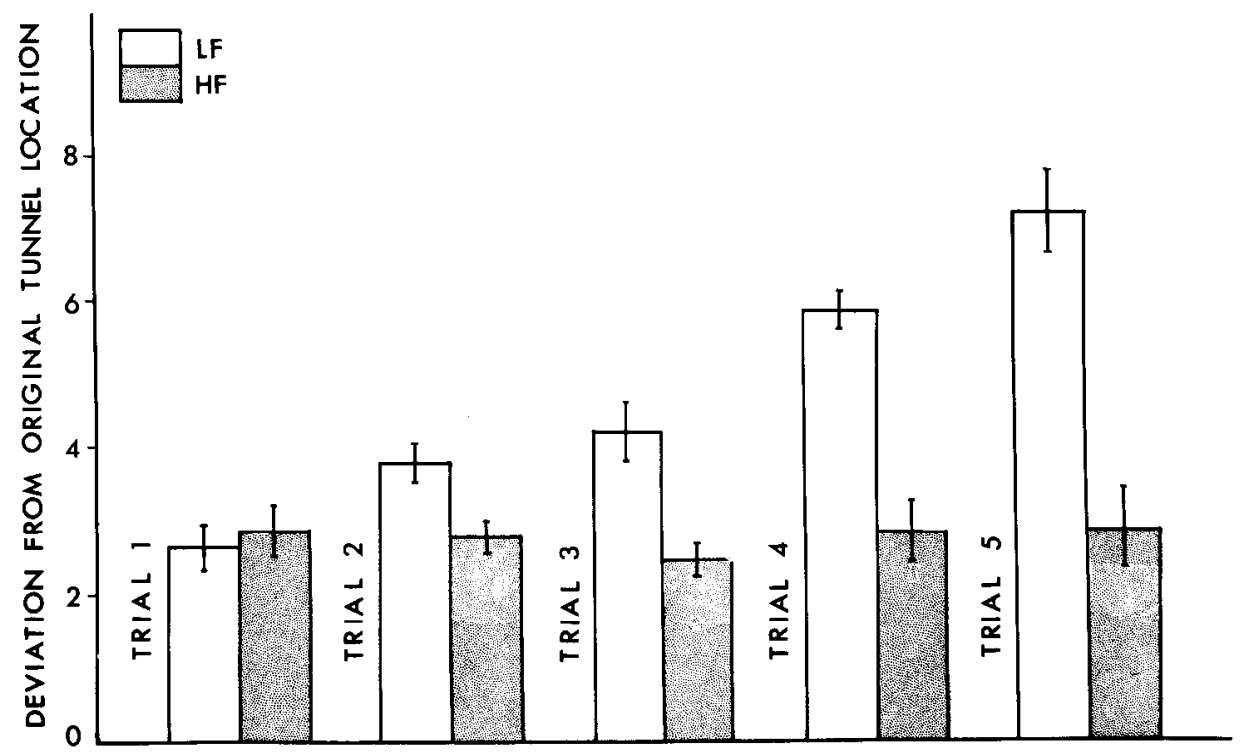




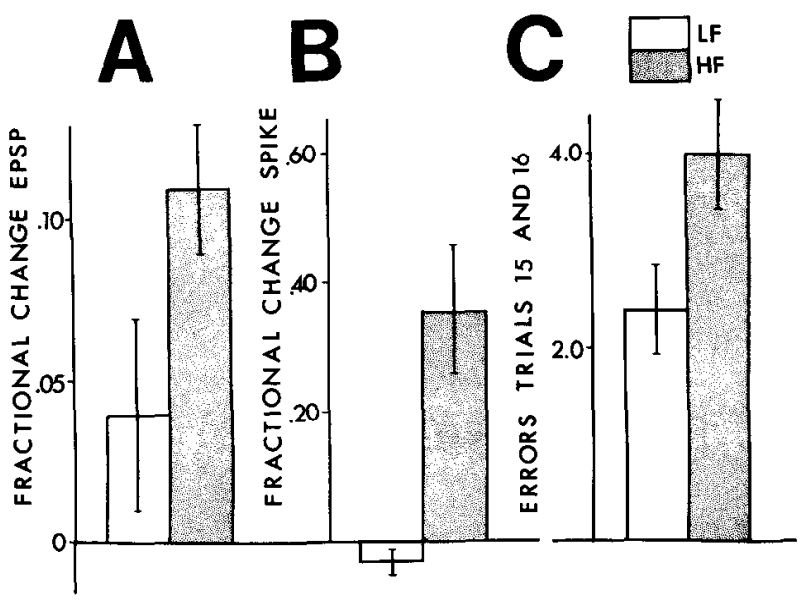

Figure 8. $A$ and $B$, LTE scores for the field EPSP and population spike in the low-frequency (LF) and high-frequency (HF) groups of Experiment 3A, measured relative to their baseline response amplitudes. The measures shown were recorded $24 \mathrm{hr}$ following the delivery of LTEinducing stimulation to group $\mathrm{HF}$, and control low-frequency stimulation to group LF. $C$, Effect of pretraining LTE-inducing stimulation on performance of the circular platform task, with a fixed tunnel location. Pooled error scores for Trials 15 and 16 showed a significant impairment of final performance in the HF group.

days (six sessions total). The animals were then retested on the radial maze for a further $3 \mathrm{~d}$. Significant LTE was observed in both response components (see Fig. 10A). There was no significant effect, however, in the mean number of errors in the three post-high-frequency radial-maze scssions, as compared with the three sessions immediately prior to high-frequency stimulation (Fig. 10B).

\section{Discussion}

The main hypothesis underlying the experiments just described was that spatial information is processed at the terminals of the primary cortical afferent pathway to the hippocampus, and stored in the form of a specific distribution of strengths of these modifiable synapses. This hypothesis led to the prediction that the induction of LTE at a large proportion of these terminals
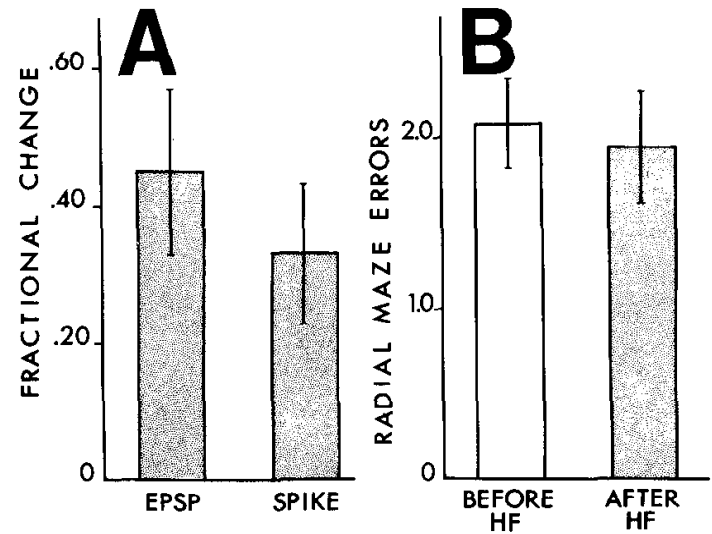

Figure 10. A, Fractional increase in field EPSP and population spike measured $24 \mathrm{hr}$ after delivery of high-frequency (HF) stimulation in Experiment 4. $B$, Working memory errors on radial eight-arm maze, averaged over $3 \mathrm{~d}$ immediately before LTE induction and for $3 \mathrm{~d}$ after HF.

would result both in a retrograde disruption of previously stored spatial information, and in an anterograde reduction in the ability to store new spatial information. These predictions were only partly verified by the results. LTE-inducing stimulation led to a profound impairment, both in the acquisition of new spatial information and in the ability to adjust adaptively to changes in previously stored spatial relationships. There was also a significant disruption of recently acquired information. The same stimulation, however, had no effect on the performance of either a well-lcarncd spatial-reference memory problem or a spatial working memory problem in which the reference components had been well-learned.

The pattern of results on the spatial-reference memory task (circular platform) is consistent with several theories of hippocampal function that postulate a role in consolidation. Such theories may be divided according to whether the role is thought to be one of temporary information storage, prior to relocation into more permanent memory (e.g., Marr, 1971) or one of nonspecific facilitation of the transfer from a short-term to a longterm store, located elsewhere, without actual information stor-

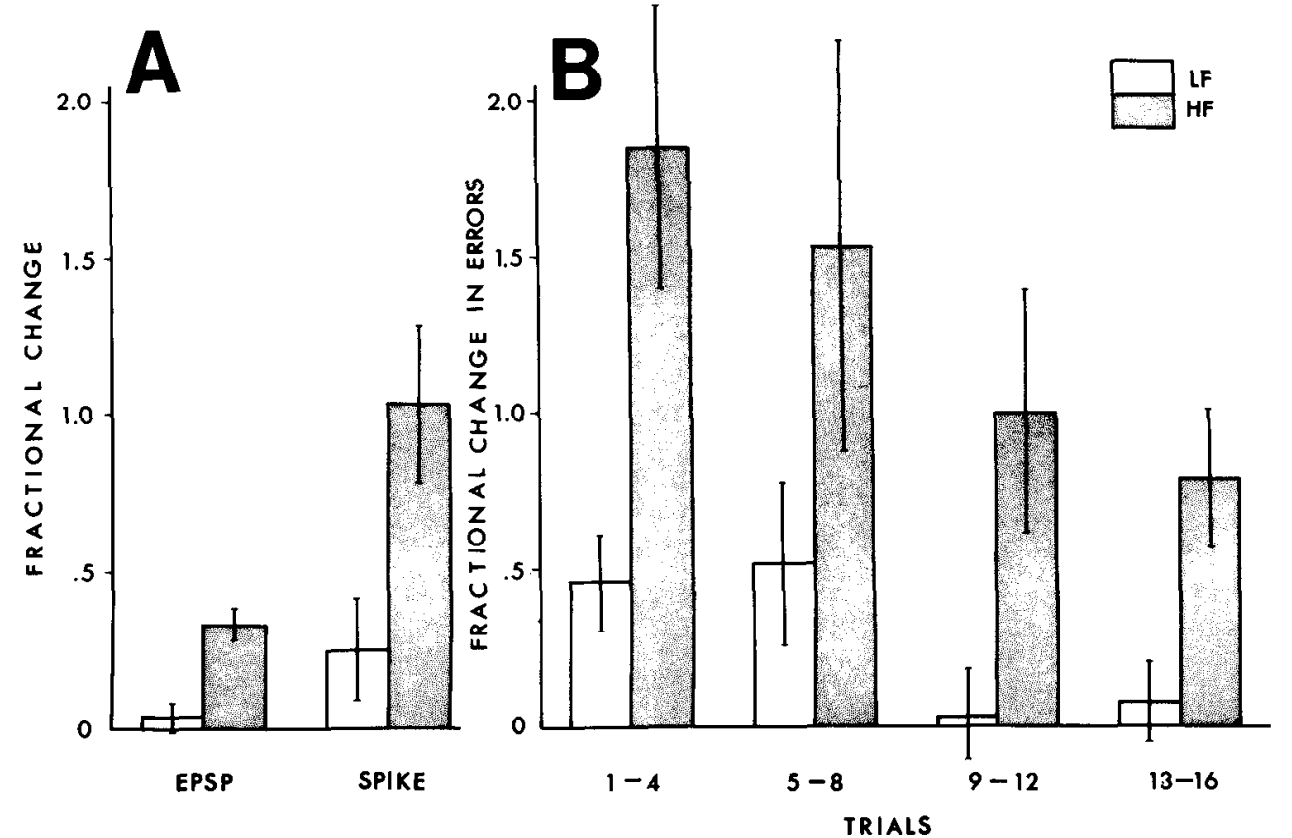

Figure 9. A, LTE scores for the field EPSP and population spike in the lowfrequency (LF) and high-frequency (HF) groups of Experiment 3B, measured relative to their baseline response amplitudes. The measures shown were recorded $24 \mathrm{hr}$ following the delivery of LTE-inducing stimulation to group HF, and control lowfrequency stimulation to group LF. $B$, Effects of high-frequency stimulation on the acquisition of new spatial information in a task that had been welllearned in a different environment. Each animal was assessed relative to its asymptotic performance of the task in the original environment. The LTEinducing stimulation produced severe impairment in the HF group. These data indicate that the spatial component of this memory problem is disrupted. 
age in the hippocampus itself (Squire et al., 1984). The present results tend to favor the former variant of the hypothesis, since it appears that the specific distribution of synaptic strengths at these hippocampal synapses is important. The nonspecific hypothesis might predict a facilitation of such a transfer, since the overall synaptic efficacy of the system was increased.

In terms of the cognitive mapping theory (O'Keefe and Nadel, 1978), these data suggest that information stored in the hippocampus is crucial for the acquisition of such a map, but not for the retention and use of a well-learned map. An alternative argument might be that the information used for the construction of cognitive maps continues to be stored in the hippocampal formation in some form other than the distribution of the weights of existing synapses (e.g., rearrangement of connections or formation of new ones).

A major dissociation exists in the results between the effects of LTE-inducing stimulation on the acquisition of information into reference memory and on acquisition of information into working memory, in a situation where the reference memory components of the task are well established. These results clearly indicate that either the nature or the location of the informationstorage mechanisms for these two types of memory are different.

The effects of LTE-inducing stimulation are at variance with the overall pattern of deficits that results from damage to all or part of the hippocampal formation. Removal of, or damage to, the entire hippocampus, entorhinal cortex, or fimbria/fornix produces large deficits in spatial working memory, spatial-reference learning and memory, and reversal behavior on preoperatively learned reference memory problems (Morris et al., 1982; Olton et al., 1978; Sutherland et al., 1983). Colchicineinduced lesions, which are thought to have selectively damaged the fascia dentata and are hence more comparable to the present treatment, produced deficits in both spatial memory and spatial acquisition, but had no effect on working memory (Jarrard, 1983; Jarrard et al., 1984). These results are thus comparable to those presented here in terms of the lack of effect on acquisition of spatial information by working memory, while spatialreference memory acquisition was disrupted. On the other hand, they differ from the present results in that the lesion experiments resulted in disruption of previously established spatial memory, while LTE-inducing stimulation appeared to leave established memories intact.

A possible interpretation of the lesion experiments is that abnormal electrical activity in the nervous system may be rather widespread following large insults to the brain. In the experiments described by Jarrard, for example (Jarrard, 1983; Jarrard et al., 1984), a number of animals in both chemical lesion and surgical aspiration groups had behaviorally manifested epileptic seizures. Since no electrophysiological recordings have been made in any of the lesion studies, it is difficult to say to what extent the activity (and, hence, information-processing capability) in the remaining brain tissue is normal. At no time in the present experiments were there signs of behavioral seizures, nor was there epileptiform activity in the EEG recorded during perforant path responsc testing sessions.

The present results also stand in contrast to those of Berger (1984) on the effects of LTE-inducing stimulation on the acquisition of the conditioned nictitating membrane response in rabbits. Berger found that LTE-inducing stimulation, delivered in a manner very similar to the method cmployed in the present experiments, facilitated the acquisition of the conditioned response. We would interpret this discrepancy as indicating that, if the hippocampus is involved in conditioned response acquisition, its role is of a permissive, rather than of an informative, nature. On the other hand, the present results lead to the conclusion that acquisition of spatial-reference memory requires the integrity of information stored in the synaptic weighting distribution of perforant path terminals. It must be recognized, however, that both Berger's and our own experiments are based on the assumption that the behavioral effects are due to the observed alterations in hippocampal synaptic efficacy, and not to some other unknown effect of high-frequency stimulation. While we can, at least, rule out number and intensity of stimulus pulses as the critical determinant, studies need to be performed in which LTE is specifically blocked during the high frequency, so that the same high-frequency stimulation can be given without the resultant change in hippocampal synaptic efficacy and behavior.

While we cannot be certain whether the effects observed here are solely due to the LTE per se, there have been a large number of studies examining the effects on learning and memory of epileptogenic or nonepileptogenic direct stimulation of the hippocampus and its associated structures. The general pattern of results has been either an improvement in performance of memory tasks (e.g., Erickson and Patel, 1969; Landfield et al., 1973; Stein and Chorover, 1968) or a retrograde impairment of recent memory (e.g., Collier et al., 1982; Kesner and Conner, 1974; Olton and Wolf, 1981; Zornetzer et al., 1973). In none of the studies cited has an acquisition impairment with a persistence corresponding to the persistence of LTE been observed. In the present experiments, anterograde disruption was observed for at least $16 \mathrm{~d}$ following the induction of LTE, while stimulation with the same number of pulses at frequencies that do not produce LTE resulted in no impairment. This strongly suggests that the independent variable in the present study corresponds to LTE itself, rather than to some other, undetermined effect of the LTE-inducing stimulation.

\section{References}

Andersen, P., S. H. Sundberg, O. Sveen, and H. Wigstrom (1977) Specific long-lasting potentiation of synaptic transmission in hippocampal slices. Nature 266: 736-737.

Barnes, C. A. (1979) Memory deficits associated with senescence: A neurophysiological and behavioral study in the rat. J. Comp. Physiol. Psychol. 93: 74-104.

Barnes, C. A., and B. L. McNaughton (1980) Spatial memory and hippocampal synaptic plasticity in senescent and middle-aged rats. In Psychobiology of Aging: Problems and Perspectives, D. O. Stein, ed., pp. 253-272, Elsevier, Amsterdam.

Barnes, C. A., and B. L. McNaughton (1983) Where is the cognitive map? Soc. Neurosci. Abstr. 10: 649.

Barnes, C. A., and B. L. McNaughton (1985a) An age comparison of the rates of acquisition and forgetting of spatial information in relation to long-term enhancement of hippocampal synapses. Behav. Neurosci. 99: 1040-1048.

Barnes, C. A., and B. L. McNaughton (1985b) In Memory Systems of the Brain: Animal and Human Cognitive Processes, N. M. Weinberger, J. L. McGaugh, and G. Lynch, eds., pp. 49-61, Guilford, New York.

Berger, T. W. (1984) Long-term potentiation of hippocampal synaptic transmission affects rate of behavioral learning. Science 224: 627630.

Birks, R. I. (1977) A long-lasting potentiation of transmitter release related to an increase in transmitter stores in a sympathetic ganglion. J. Physiol. (Lond.) 27 1: 847-862.

Bliss, T. V. P., and A. Gardner-Medwin (1973) Long-lasting potentiation of synaptic transmission in the dentate area of the unanesthetized rabbit following stimulation of the perforant path. J. Physiol. (Lond.) 232: 357-374.

Bliss, T. V. P., and T. Lomo (1973) Long-lasting potentiation of synaptic transmission in the dentate area of the anesthetized rabbit following stimulation of the perforant path. J. Physiol. (Lond.) 232:331356.

Collier, T. J., J. S. Miller, J. Travis, and A. Routtenberg (1982) Dentate gyrus granule cells and memory: Electrical stimulation disrupts memory for places rewarded. Behav. Neural Biol. 34: 227-239.

Del Castillo, J., and B. Katz (1954) Statistical factors involved in neuromuscular facilitation and depression. J. Physiol. (Lond.) 124: 574-585.

Erickson, C. K., and J. B. Patel (1969) Facilitation of avoidance learn- 
ing by posttrial hippocampal electrical stimulation. J. Comp. Physiol. Psychol. 68: 400-406.

Gabriel, M., S. P. Sparenborg, and N. Stolar (in press) In The Hippocampus, Vol. III, R. L. Isaacson and K. H. Pribram, eds., Plenum, New York.

Hebb, D. O. (1949) The Organization of Behavior, Wiley-Interscience, New York.

Jarrard, L. E. (1983) Selective hippocampal lesions and behavior: Effects of kainic acid lesions on performance of place and cue tasks. Bchav. Neurosci. 97: 873-889.

Jarrard, L. E., O. Hiroshige, O. Steward, and R. B. Goldschmidt (1984) On the role of hippocampal connections in the performance of place and cue tasks: Comparisons with damage to hippocampus. Behav. Neurosci. 98: 946-954.

Kesner, R. P., and H. S. Conner (1974) Effects of electrical stimulation of rat limbic system and midbrain reticular formation upon shortand long-term memory. Physiol. Behav. 12:5-12.

Kubie, J. L., and J. B. Ranck, Jr. (1983) Sensory-behavioral correlates in individual hippocampus neurons in three situations: Space and context. In Neurobiology of the Hippocampus, W. Seifert, ed., pp. 433-447, Academic, London.

Landfield, P. W., R. J. Tusa, and J. L. McGaugh (1973) Effects of posttrial hippocampal stimulation on memory storage and EEG activity. Bchav. Biol. 8: 485-505.

Levy, W. B., and O. Steward (1979) Synapses as associative memory elements in the hippocampal formation. Brain Res. 175: 233-245.

Liley, A. W., and K. A. K. North (1953) An electrical investigation of effects of repetitive stimulation on mammalian neuromuscular junction. J. Neurophysiol. 16: 509-527.

Lloyd, D. (1949) Post-tetanic potentiation of response in monosynaptic reflect pathways of the spinal cord. J. Gen. Physiol. 33: 147170.

Lomo, T. (1966) Frequency potentiation of excitatory synaptic activity in the dentate area of the hippocampal formation. Acta Physiol. Scand. 68: 128

Lomo, T. (1971) Potentiation of monosynaptic EPSP's in the perforant path-dentate granule cell synapse. Exp. Brain Res. 12: 46-63.

Maglcby, K. L., and J. E. Zengel (1975) A quantitative description of tetanic and post-tetanic potentiation of transmitter release at the frog neuromuscular junction. J. Physiol. (Lond.) 245: 183-208.

Marr, D. (1971) Simple memory: A theory of archicortex. Phil. Trans. R. Soc. [Biol.] 262: 23-80.

McNaughton, B. L. (1980) Evidence for two physiologically distinct perforant pathways to the fascia dentata. Brain Res. 199: 1-19.

McNaughton, B. L. (1983) Activity dependent modulation of hippocampal synaptic efficacy: Some implications for memory processes. In The Neurobiology of the Hippocampus, W. Seifert, ed., pp. 233251, Academic, London.

McNaughton, B. L., and C. A. Barnes (1977) Physiological identification and analysis of dentate granule cell responses to stimulation of the medial and lateral perforant pathways in the rat. J. Comp. Neurol. 175: 439-454.

McNaughton, B. L., C. A. Barnes, and J. O'Keefe (1983) The contributions of position, direction, and velocity to single unit activity in the hippocampus of freely-moving rats. Exp. Brain Res. 52: 41-49.

McNaughton, B. L., R. M. Douglas, and G. V. Goddard (1978) Synaptic enhancement in fascia dentata: Cooperativity among coactive afferents. Brain Res. 157: 277-293.

Molino, A., and D. McIntyre (1972) Another inexpensive headplug for the electrical recording and/or stimulation of rats. Physiol. Behav. 9: $273-275$.
Morris, R. G. M., P. Garrud, J. N. P. Rawlins, and J. O'Keefe (1982) Place navigation impaired in rats with hippocampal lesions. Nature 297: 681-683

O'Keefe, J. (1976) Place units in the hippocampus of the freely moving rat. Exp. Neurol. 51: 78-109.

O'Keefe, J., and J. Dostrovsky (1971) The hippocampus as a spatial map. Preliminary evidence from unit activity in the freely-moving rat. Brain Res. 34: 171-175.

O'Keefe, J., and L. Nadel (1978) The Hippocampus as a Cognitive Map, Oxford, New York.

Olton, D. S. (1983) Memory functions in the hippocampus. In The Neurobiology of the Hippocampus, W. Seifert, ed., pp. 335-373, Academic, London.

Olton, D. S., and R. J. Samuelson (1976) Remembrance of places passed: Spatial memory in rats. J. Exp. Psychol. Anim. Behav. Processes 2: 97-116

Olton, D. S., and W. A. Wolf (1981) Hippocampal seizures produce retrograde amnesia without a temporal gradient when they reset working memory. Behav. Neural Biol. 33: 437-452.

Olton, D. S., J. T. Becker, and G. E. Handelmann (1979) Hippocampus, space, and memory. Behav. Brain Sci. 2: 313-365.

Olton, D. S., M. Branch, and P. J. Best (1978) Spatial correlates of hippocampal unit activity. Exp. Neurol. 58: 387-409.

Olton, D. S., J. A. Walker, and F. H. Gage (1978) Hippocampal connections and spatial discrimination. Brain Res. 139: 295-308.

Ranck, Jr., J. B. (1984) Head direction cells in the deep cell layer of dorsal presubiculum in freely moving rats. Soc. Neurosci. Abstr. 10: 599.

Rao, G., B. L. McNaughton, and C. A. Barnes (1984) Retrograde spatial amnesia gradient following hippocampal LTE. Soc. Neurosci. Abstr. 10: 126.

Rawlins, J. N. P. (1985) Associations across time: The hippocampus as a temporary memory store. Behav. Brain Sci. 8: 479-496.

Sharp, P. E., B. L. McNaughton, and C. A. Barnes (1985) Enhancement of hippocampal field potentials in rats exposed to a novel, complex environment. Brain Res. 339: 361-365.

Squire, L. R., and N. J. Cohen (1984) Human memory and amnesia. In Neurobiology of Learning and Memory, G. Lynch, J. L. McGaugh, and N. M. Weinberger, eds., pp. 3-64, Guilford, New York.

Squire, L. R., N. J. Cohen, and L. Nadel (1984) The medial temporal region and memory consolidation: A new hypothesis. In Memory Consolidation, H. Weingartner and E. Parker, eds., pp. 185-210, Erlbaum, Hillsdale, NJ.

Stein, D. G., and S. L. Chorover (1968) Effects of posttrial electrical stimulation of hippocampus and caudate nucleus on maze learning in the rat. Physiol. Behav. 3: 787-791.

Sutherland, R. J., I. Q. Whishaw, and B. Kolb (1983) A behavioral analysis of spatial localization following electrolytic, kainate-, or colchicine-induced damage. Behav. Brain Res. 7: 133-153.

Swanson, L. W., T. J. Teyler, and R. F. Thompson (1982) Hippocampal long-term potentiation: Mechanisms and implications for memory. Neurosci. Res. Prog. Bull. 20: 613-769.

Teyler, T. J., and P. Discenna (1984) Long-term potentiation as a candidate mnemonic device. Brain Res. Rev. 7: 15-28.

Teyler, T. J., and P. Discenna (in press) The hippocampal memory indexing theory. Behav. Neurosci.

Zornetzer, S. F., R. B. Chronister, and B. Ross (1973) The hippocampus and retrograde amnesia: Localization of some positive and negative memory disruptive sites. Behav. Biol. 8: 507-518. 